\title{
Configural Weighting in Judgments of Two- and Four-Outcome Gambles
}

\author{
Michael H. Birnbaum \\ California State University, Fullerton, \\ and the Institute for Mathematical Behavioral Sciences
}

\author{
Richard Veira \\ California State University, Fullerton
}

\begin{abstract}
This study tested branch independence, a key property distinguishing nonconfigural from configural theories of decision making. Sixty undergraduates judged buying and selling prices of 168 lotteries composed of 2 or 4 equally likely outcomes, $(x, y, z, v)$. Branch independence requires that $(x, y, z, v)$ is judged higher than $\left(x^{\prime}, y^{\prime}, z, v\right)$ whenever $\left(x, y, z^{\prime}, v^{\prime}\right)$ is judged higher than $\left(x^{\prime}, y^{\prime}, z^{\prime}, v^{\prime}\right)$. Different violations observed in different viewpoints are consistent with the theory that the utility function is independent of viewpoint and that only configural weights differ between viewpoints. Lower ranked outcomes have greater weights in the buyer's than in the seller's viewpoint. Sellers place more weight than buyers on higher ranked outcomes. In both viewpoints, violations of branch independence are contrary to the inverse-S weighting function of cumulative prospect theory: Moderate outcomes receive more weight than adjacent extreme outcomes.
\end{abstract}

This study tests a key distinction between a class of subjective expected utility (SEU) models (Savage, 1954) and configural weight models (Birnbaum \& Beeghley, 1997; Birnbaum \& McIntosh, 1996). SEU theory and other nonconfigural models imply branch independence, a weaker form of Savage's "sure thing" principle. Branch independence requires that if two gambles have one or more common branches (the same outcome produced by the same event with the same known probability), then the preference order induced by other components of gambles will be independent of the value of the common outcome(s).

Let $(x, y, z)$ denote a gamble in which the outcomes $x, y$, and $z$ are equally likely. Branch independence requires that $(x, y, z)$ is judged better than $\left(x^{\prime}, y^{\prime}, z\right)$ if and only if $\left(x, y, z^{\prime}\right)$ is judged better than $\left(x^{\prime}, y^{\prime}, z^{\prime}\right)$. Birnbaum and McIntosh (1996) found that most judges prefer $(\$ 40, \$ 44, \$ 2)$ over $(\$ 10, \$ 98$, $\$ 2$ ); however, most judges preferred $(\$ 10, \$ 98, \$ 108)$ over $(\$ 40, \$ 44, \$ 108)$, violating branch independence. Birnbaum and Beeghley (1997) found similar results with judgments of value from both the buyer's and the seller's points of view, indicating that violations of branch independence are not produced by a process unique to either choice or judgment. Instead, the results suggest that the violations are produced by an aspect of evaluation of gambles that is common to all three tasks (choices, buying prices, and selling prices).

Michael H. Birnbaum, Department of Psychology, California State University, Fullerton, and the Institute for Mathematical Behavioral Sciences, Irvine, California; Richard Veira, Department of Psychology, California State University, Fullerton.

Support was received from National Science Foundation Grant SBR-9410572 through California State University, Fullerton. We thank Jonathan Baron, R. Duncan Luce, and Mary Kay Stevenson for their helpful comments on a draft of this article.

Correspondence concerning this article should be addressed to Michael H. Birnbaum, Department of Psychology, CSUF H-830M, California State University, P.O. Box 6846, Fullerton, California 92834-6846. Electronic mail may be sent via Internet to mbirnbaum@fullerton.edu.
Violations of branch independence in these studies are inconsistent with expected utility (EU) and SEU formulations, including psychological generalizations that allow a weighting function of probability (e.g., Edwards, 1954). Systematic violations are also inconsistent with the theory that people edit and eliminate common outcomes when choosing between gambles (Kahneman \& Tversky, 1979). The empirical pattern of results is also opposite that predicted by the inverse-S weighting function of cumulative prospect theory (Tversky \& Kahneman, 1992). The inverse-S weighting function implies that the middle outcome has less weight than the extreme outcomes, but the pattern of violations observed is opposite from that predicted by this type of weighting function (Birnbaum \& McIntosh, 1996).

Because these violations of branch independence contradict so many theories of decision making, it is important to determine whether these findings may be unique to the situation of three outcomes used by Birnbaum and Beeghley (1997) and Birnbaum and McIntosh (1996). Three-outcome gambles form a special case because the middle outcome is also the unique median. The intuitive description of such gambles is that there are two low outcomes and one high one or that there are one low outcome and two high ones, so the middle of three outcomes might have a special psychological role in forming a majority. Therefore, it is important to determine whether results observed with three-outcome gambles generalize to gambles with an even number of outcomes, such as two and four.

\section{SEU and Branch Independence}

In the present experiment, we described lotteries composed of two equally likely outcomes, denoted $(x, y)$, and four equally likely outcomes, denoted $(x, y, z, v)$ as follows: For four-outcome gambles, a can contains four slips that are identical with the exception that different numbers are written on them. The slips will be mixed, and one will be drawn blindly at random to determine the outcome. If slip 1 were chosen, the outcome would be $x$; if slip 2 were chosen, 
the outcome would be $y$; if slip 3 were chosen, the outcome would be $z$; and if slip 4 were chosen, the outcome would be $v$. With two-outcome gambles, there are two equally likely slips.

The SEU for such a four-outcome gamble can be written as follows:

$$
\begin{aligned}
& \operatorname{SEU}(x, y, z, v)= \\
& \quad s(p) u(x)+s(p) u(y)+s(p) u(z)+s(p) u(v),
\end{aligned}
$$

where $\operatorname{SEU}(x, y, z, v)$ represents the subjective expected utility of the gamble; $s(p)$ is the weight of $p=1 / 4$ in this case, and $u(x), u(y), u(z)$, and $u(v)$ represent the subjective values of the monetary outcomes. In Savage's (1954) theory, $s(p)=1 / 4$; however, Edwards' (1954) psychological generalization allows $s(p)$ to differ from $p$. When $s(p)$ does not equal $p$, Equation 1 is sometimes called subjectively weighted utility theory.

For judgments of four-outcome gambles with two common outcomes, branch independence can be written as follows:

$$
\begin{gathered}
(x, y, z, v) \text { is judged higher than }\left(x^{\prime}, y^{\prime}, z, v\right) \\
\text { if and only if } \\
\left(x, y, z^{\prime}, v^{\prime}\right) \text { is judged higher than }\left(x^{\prime}, y^{\prime}, z^{\prime} v^{\prime}\right) .
\end{gathered}
$$

In other words, replacing the common outcomes $(z, v)$ with $\left(z^{\prime}, v^{\prime}\right)$ should not affect the direction of preference between $(x, y)$ and $\left(x^{\prime}, y^{\prime}\right)$. In this restricted case, with a fixed number of distinct outcomes at fixed probabilities, branch independence is equivalent to joint independence (Krantz, Luce, Suppes, \& Tversky, 1971, p. 339).

SEU theory implies that the terms involving $z$ and $v$ can be subtracted off both sides of the inequality and that terms with $z^{\prime}$ and $v^{\prime}$ can be added to both sides of the inequality representing a preference (Birnbaum \& Beeghley, 1997). Therefore, both classic SEU theory and Edwards' (1954) psychological version of SEU theory (Equation 1) imply branch independence (Expression 2) in an experiment with a fixed number of distinct, equally probable values.

\section{Rank-Dependent Configural Weighting}

In contrast, rank-dependent utility (RDU) theory (e.g., Luce, 1992) allows different weights for outcomes of different ranks (see also Birnbaum, 1974a, p. 559). For this experiment, a rank-dependent, configural weight model can be written as follows:

$$
\begin{aligned}
\operatorname{RDU}(x, y, z, v) & = \\
& w_{\mathrm{L}} u(x)+w_{\mathrm{ML}} u(y)+w_{\mathrm{MH}} u(z)+w_{\mathrm{H}} u(v)
\end{aligned}
$$

for $0<x<y<z<v$, where $w_{L}, w_{M L}, w_{M H}$, and $w_{H}$ are the configural weights of the lowest, medium-low, mediumhigh, and highest outcomes (at equal probabilities of 1/4), respectively. If the judged value of a gamble is a monotonic function of the RDU, then the model can be written as follows:

$\mathrm{P}(x, y, z, v)=$

$$
\mathrm{J}\left[w_{\mathrm{L}} u(x)+w_{\mathrm{ML}} u(y)+w_{\mathrm{MH}} u(z)+w_{\mathrm{H}} u(v)\right],
$$

where $P$ is the predicted judgment of price and $\mathbf{J}$ is a strictly monotonic judgment function that converts utilities into monetary judgments. Note that a nonlinear judgment function cannot produce or eliminate violations of branch independence, which is a purely ordinal property.

Equations 3 and 4 imply violations of branch independence (see Birnbaum \& McIntosh, 1996). For example, if outcomes such that $0<z^{\prime}<v^{\prime}<x^{\prime}<x<y<y^{\prime}<z<v$ are selected, then the following pattern of violations of branch independence occurs:

$\mathrm{P}\left(z^{\prime}, v^{\prime}, x, y\right)>\mathrm{P}\left(z^{\prime}, v^{\prime}, x^{\prime}, y^{\prime}\right)$

$$
\text { and } \mathrm{P}(x, y, z, v)<\mathrm{P}\left(x^{\prime}, y^{\prime}, z, v\right)
$$

if and only if

$$
\frac{w_{\mathrm{MH}}}{w_{\mathrm{H}}}>\frac{u\left(y^{\prime}\right)-u(y)}{u(x)-u\left(x^{\prime}\right)}>\frac{w_{\mathrm{L}}}{w_{\mathrm{ML}}} .
$$

The opposite pattern of violations of branch independence,

$$
\begin{aligned}
& \mathrm{P}\left(z^{\prime}, v^{\prime}, x, y\right)<\mathrm{P}\left(z^{\prime}, v^{\prime}, x^{\prime}, y^{\prime}\right) \\
& \text { and } \mathrm{P}(x, y, z, v)>\mathrm{P}\left(x^{\prime}, y^{\prime}, z, v\right),
\end{aligned}
$$

occurs if and only if

$$
\frac{w_{\mathrm{MH}}}{w_{\mathrm{H}}}<\frac{u\left(y^{\prime}\right)-u(y)}{u(x)-u\left(x^{\prime}\right)}<\frac{w_{\mathrm{L}}}{w_{\mathrm{ML}}} .
$$

Expressions 5a-b and $6 \mathrm{a}-\mathrm{b}$ show that if the ratios of weights are equal, there will be no violations of branch independence between the cases in which the common outcomes are changed from lowest to highest. Because EU and SEU theories assume that weights are independent of rank, these ratios are all one, and there should therefore be no systematic violations of branch independence in any experiment. To observe a violation in a finite experiment of this type, one must use values of the outcomes such that the ratio of differences of utility is straddled by the ratios of weights, as in Expressions 5b or 6b.

\section{Point of View, Configural Weighting, and Scale Convergence}

Birnbaum and Stegner (1979) defined "point of view" of the judge in terms of payoffs or instructions that cause the judge to have different psychological costs for overestimating or underestimating when forming a judgment of value. 
This definition is analogous to the manipulation of payoffs in signal detection experiments that differentially affect the costs of false alarms and rewards for hits. Bimbaum and Stegner found that the rank order of judgments can be changed by manipulation of the judge's point of view (see also Birnbaum, 1982).

When configural weight theory has been fit to judgments of uncertain and risky prospects, configural weights depend on the judge's point of view. In the buyer's viewpoint, more weight is placed on lower valued outcomes or estimates of value; in the seller's point of view, relatively more weight is placed on higher and middle values compared with the buyer's point of view (Birnbaum \& Beeghley, 1997; Birnbaum, Coffey, Mellers, \& Weiss, 1992; Birnbaum \& Stegner, 1979; Birnbaum \& Sutton, 1992; Birnbaum \& Zimmermann, 1995). These studies have also found that $u(x)$ can be approximated by $u(x)=x$, for $0<x<\$ 150$.

The term scale convergence refers to the assumption that the utility (or value) functions are independent of point of view and task (Birnbaum, 1974a; Birnbaum \& Sutton, 1992). With the same utility function, $u(x)=x$, the configural weight model can account for reversals of preference found in judgment and choice (Birnbaum \& Beeghley, 1997; Birnbaum \& McIntosh, 1996).

In studies of violations of branch independence in threeoutcome gambles, it has been found that the preference order is consistent with

$$
\frac{w_{\mathrm{M}}}{w_{\mathrm{H}}}>\frac{w_{\mathrm{L}}}{w_{\mathrm{M}}}
$$

where $w_{L}, w_{M}$, and $w_{H}$ are the configural weights of the lowest, middle, and highest of three, equally likely, positive outcomes. Thus, the middle outcome has greater weight than the geometric average of the extremes (Equation $7 \Leftrightarrow w_{\mathrm{M}}>$ $\left.\sqrt{w_{\mathrm{L}} w_{\mathrm{M}}}\right)$. This pattern was observed in both the buyer's and the seller's points of view (Birnbaum \& Beeghley, 1997) and in choice experiments (Birnbaum \& McIntosh, 1996). Extrapolating to four outcomes, this finding suggests that Equations $5 \mathrm{a}$ and $5 \mathrm{~b}$ rather than Equations $6 \mathrm{a}$ and $6 \mathrm{~b}$ might be descriptive of judgments. This pattern is opposite to the pattern predicted by the inverse-S weighting function used in the model of cumulative prospect theory (Tversky \& Kahneman, 1992; Wu \& Gonzalez, 1996).

\section{Cumulative Weighting Functions}

Cumulative prospect theory is a rank- and sign-dependent utility theory similar to that of Luce and Fishburn (1991, 1995). In the cumulative prospect model of Tversky and Kahneman (1992), the decumulative weighting function for positive outcomes is further specified to follow an inverse-S function of decumulative probability (see also Tversky \& Wakker, 1995; Wu \& Gonzalez, 1996). Outcomes are ranked in decreasing value, $x_{1}>x_{2}>x_{3}>\ldots$; weights of the ranked outcomes are assumed to follow the expression

$$
w_{i}=W\left(p_{i}\right)-W\left(p_{i-1}\right),
$$

where $w_{i}$ is the weight of outcome, $x_{\mathrm{i}}, p_{\mathrm{i}}$ is the (decumulative) probability that an outcome is greater than or equal to $x_{i}$ given the gamble, and $p_{i-1}$ is the probability that the outcome is strictly greater than $x_{i}$ (which is the same as the probability that the outcome is greater than or equal to the next higher value, $\left.x_{i-1}\right)$. The function $W$ also satisfies $W(0)=0$ and $W(1)=1$.

For four-outcome gambles, the cumulative prospect model is a special case of Equation 3, constrained to follow Equation 8a, and also assumed to follow an inverse-S decumulative weighting function. The decumulative weighting function fit by Tversky and Kahneman (1992) is as follows:

$$
W(p)=\frac{p^{\gamma}}{\left[p^{\gamma}+(1-p)^{\gamma}\right]^{1 / \gamma}},
$$

where $\gamma$ is the parameter of the weighting function, estimated to be 0.61 . This decumulative weighting function implies that four equally likely outcomes will have weights of $0.43,0.15,0.13$, and 0.29 for $w_{L}, w_{\mathrm{ML}}, w_{\mathrm{MH}}$, and $w_{\mathrm{H}}$, respectively. Because less weight is assigned to middle outcomes than to extreme ones, this inverse-S weighting function implies Expression $6 \mathrm{~b}$ and, therefore, the preference order of Expression 6a. These weights are shown in Figure 1B for comparison with the equal weights of SEU theory, which are shown in Figure 1A.

Any inverse-S weighting function assigns less weight to medium outcomes than to extreme outcomes; therefore, it makes the same implication for tests of branch independence. If $w_{\mathrm{MH}}<w_{\mathrm{H}}$, then $w_{\mathrm{MH}} / w_{\mathrm{H}}<1$, and if $w_{\mathrm{ML}}<w_{\mathrm{L}}$, then $1<w_{\mathrm{L}} / w_{\mathrm{ML}}$; therefore, $w_{\mathrm{MH}} / w_{\mathrm{H}}<w_{\mathrm{L}} / w_{\mathrm{ML}}$, as in Expression $6 \mathrm{~b}$. Any such inverse-S weighting function therefore implies the preference pattern in Expression 6a, whereas an S-shaped weighting function (more weight on medium outcomes) would imply the opposite. This study tests between these implications.

Luce and Fishburn $(1991,1995)$ give arguments that the decumulative weighting function might be a power function of probability, instead of the inverse-S of Expression 8b. Consider a binary gamble with a probability $q$ to win a chance at another (independent) gamble or otherwise receive 0 , and suppose the second gamble has probability $p$ to win $y$, or otherwise 0 . If the utility of such a gamble, $\mathrm{U}(y, p, 0)$, can be represented by the product $u(y) W(p)$, then a power function,

$$
W(p)=p^{\gamma},
$$

preserves the rational multiplication of independent probabilities, $\mathrm{U}[(y, p, 0), q, 0] \sim \mathrm{U}[y, p q, 0]$.

If $\gamma>1$, this weighting scheme (Equations 8a and 8c) can imply the preference pattern dictated by Expression $5 b$, and with $\gamma<1$, it can imply Expression $6 \mathrm{~b}$. For example, with $\gamma=1.5$, the weights are $0.350,0.296,0.229$, and 0.125 for $w_{\mathrm{L}}, w_{\mathrm{ML}}, w_{\mathrm{MH}}$, and $w_{\mathrm{H}}$, respectively. These weights, shown in Figure 1C, satisfy Expression $5 \mathrm{~b}$ and, therefore, imply the pattern of Expression 5a.

Assuming Expression 8a, the power function (Equation 8c) implies that weights of the ranks are a monotonic 


\section{A. SEU Welghts}

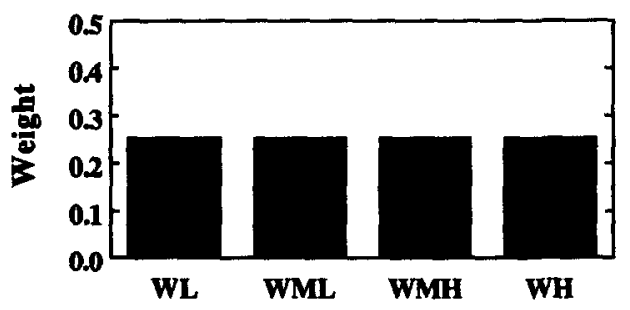

B. Inverse-S Weighting of CPT

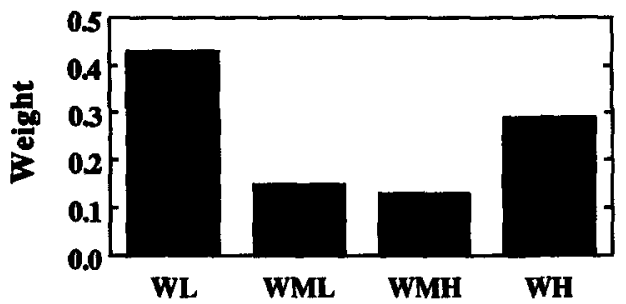

C. Power Weighting Function

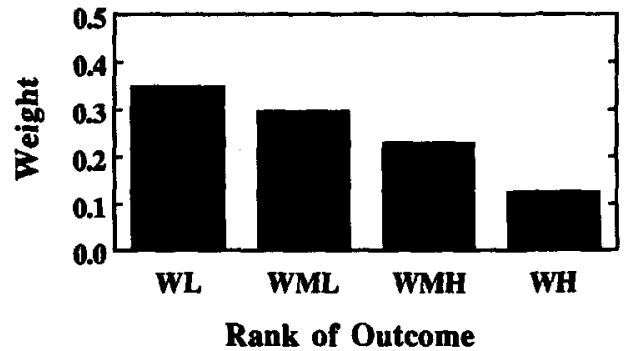

Figure 1. Weights of outcomes as a function of rank according to different theories. (A) subjective expected utility (SEU) theory implies that weights of all ranks should be equal. (B) Inverse-S weighting function of cumulative prospect theory (CPT) implies that weights of middle outcomes are lower than those of extreme outcomes. (C) Power cumulative weighting function implies that weights should be a monotonic function of rank. WL, WML, WMH, and WH are the configural weights of the lowest, mediumlow, medium-high, and highest outcomes, respectively.

function of rank, or they are all equal (when $\gamma=1$ ). The power function would not be consistent with greater (or lesser) weights in the middle than at both ends. Such a pattern might be consistent instead with an S-shape (or inverse-S) decumulative weighting function, respectively. This study assesses this implication for the estimated weights, assuming $u(x)=x$.

If SEU were to hold, even if the utilities (or a reference point for the utility function) were to change for different points of view, then the preference order of the gambles could change in different viewpoints, but there should be no violations of branch independence. If rank-dependent, configural weight utility holds, however, then there can be violations of branch independence, and furthermore, if point of view affects weights, rather than just utilities or reference points, then the violations of branch independence can change in different points of view. The exact pattern of violations can distinguish different patterns of rankdependent configural weighting.

A number of articles have explored models in which the weights of stimuli are assumed or permitted to depend on the stimulus configuration (Birnbaum, 1973, 1974a, 1982; Birnbaum et al., 1992; Birnbaum \& Sotoodeh, 1991; Birnbaum \& Stegner, 1979, 1981; Birnbaum \& Sutton, 1992; Champagne \& Stevenson, 1994; Edwards, 1992; Kahneman \& Tversky, 1979; Lopes, 1990; Luce, 1992; Luce \& Fishburn, 1991, 1995; Luce \& Narens, 1985; Machina, 1982; Miyamoto, 1989; Quiggin, 1982; Schmeidler, 1989; Tversky \& Kahneman, 1992; Wakker, 1993, 1994; Wakker, Erev, \& Weber, 1994; Weber, 1994; Weber, Anderson, \& Birnbaum, 1992; Yaari, 1987). Those making specific predictions concerning the form of the weighting function can be distinguished in the present experiments.

\section{Method}

\section{Instructions}

Judges were instructed to estimate the values of gambles composed of two or four equally likely outcomes. The first paragraph described a hypothetical example of a 50-50 chance to win either $\$ 10$ or $\$ 96$, in which the outcome would be determined by drawing one of two slips from a can, and asked how much this gamble would be worth from both the buyer's and the seller's points of view.

In the buyer's point of view, judges were asked to imagine that they were "deciding the most a buyer should pay to buy the chance to play the lottery." They were told that the buyer exchanges money for the chance to play the lottery.

In the seller's point of view, judges were asked to imagine they were "deciding the least that a seller should accept to sell the lottery." They were told the seller receives money and gives up the chance to play the lottery. Additional instructions for buying and selling tasks were as given in Birnbaum and Sutton (1992).

\section{Stimuli}

Each four-outcome lottery was displayed as in the following example: $(\$ 5, \$ 24, \$ 72, \$ 96)$. This situation was described as offering one chance out of four to win $\$ 5$, one chance to win $\$ 24$, one chance to win $\$ 72$, and one chance to win $\$ 96$. The gamble was described as a can containing four otherwise identical slips with the outcomes printed on them. These would be mixed, and one would be chosen blindly and at random to determine the outcome. A two-outcome gamble would be displayed as in the following example: $(\$ 24, \$ 96)$, which was described as a can containing two equally likely slips.

\section{Design}

There were 140 gambles consisting of four equally likely outcomes $(x, y, z, v)$, constructed from a $5 \times 28$ factorial design with 5 levels of common outcomes $(z, v)$ combined with $28(x, y)$ pairs. The levels of $(z, v)$ were $(\$ 2, \$ 4),(\$ 124, \$ 148),(\$ 34, \$ 37)$, $(\$ 19, \$ 52)$, and $(\$ 2, \$ 148)$. The 28 levels of $(x, y)$ pairs were listed according to the range $(|x-y|)$, in descending order of total value $(x+y):$ range $=\$ 0:(\$ 54, \$ 54),(\$ 36, \$ 36),(\$ 24, \$ 24) ;$ range $=$ $\$ 6:(\$ 51, \$ 57),(\$ 45, \$ 51),(\$ 39, \$ 45),(\$ 33, \$ 39),(\$ 27, \$ 33),(\$ 21$, $\$ 27),(\$ 15, \$ 21)$; range $=\$ 12 ;(\$ 48, \$ 60),(\$ 42, \$ 54),(\$ 36, \$ 48)$, 
$(\$ 30, \$ 42),(\$ 24, \$ 36),(\$ 18, \$ 30),(\$ 12, \$ 24) ;$ range = \$24: $(\$ 42$, $\$ 66),(\$ 12, \$ 36) ;$ range $=\$ 36:(\$ 36, \$ 72),(\$ 12, \$ 48)$; range $=$ $\$ 48:(\$ 30, \$ 78),(\$ 12, \$ 60) ;$ range $=\$ 60:(\$ 24, \$ 84),(\$ 12, \$ 72)$; range $=\$ 72:(\$ 18, \$ 90),(\$ 12, \$ 84) ;$ range $=\$ 84:(\$ 12, \$ 96)$. Totals $(x+y)$ ranged from $\$ 36$ to $\$ 108$, in steps of $\$ 12$; there were at least two levels of range for each total.

There were also 28 gambles consisting of two-outcome gambles, which used the same $28(x, y)$ pairs, yielding a total of 168 experimental gambles.

\section{Procedure}

The 168 combinations were printed in random order in the booklets, with the restrictions that the same $(x, y)$ or $(z, v)$ pair would not be repeated on consecutive trials, nor would twooutcome gambles appear on consecutive trials. Each judge evaluated all 168 gambles from viewpoints of both buyer and seller. The buyer's and the seller's booklets were identical, with instructions that described both points of view, except that the final paragraph of instructions reiterated the task to be performed next. The final paragraph in the buyer's booklet gave another summary of the buyer's point of view, followed by eight warm-up trials for the buyer's task. The final paragraph for the seller's booklet reviewed the seller's task, followed by the same warm-up trials, to be judged from the seller's viewpoint. Half of the groups received the buyer's booklet first, followed by the seller's booklet, and half received the seller's booklet first, followed by the buyer's booklet.

The experimenter read the instructions aloud while judges followed in their booklets. Judges worked at their own paces and completed both tasks in 2 hrs.

\section{Judges}

Judges were 60 undergraduates, who participated as one option toward completing an assignment in introductory psychology.

\section{Results}

Figure 2 shows mean judgments from the buyer's point of view for $(x, y)$ and $(x, y, z, v)$ gambles for pairs in which $x+$ $y=108$, with separate symbols for each $(z, v)$ pair. Mean judgments are plotted as symbols, and predictions of a configural-weight model (discussed in the next section) are shown as solid and dashed lines. Figure 2 shows that for two-outcome gambles, shown as open circles, mean judgments decrease as $|x-y|$ increases. Similarly, for all values of $(z, v)$ except $(\$ 124, \$ 148)$, mean judgments decrease or stay level as $|x-y|$ increases. However, when the common outcomes are the highest $(\$ 124, \$ 148)$, shown as filled triangles in Figure 2, mean judgments increase. Changes in sign of the slopes for different common outcomes constitute violations of branch independence in the mean judgments.

Figure 3 shows mean judgments from the seller's point of view, plotted as in Figure 2. The mean judgments decrease as a function of $|x-y|$ for the lowest four curves. However, for $(\$ 2, \$ 148)$, shown as open squares, and for $(\$ 124, \$ 148)$, shown as filled triangles, the mean judgments increase with increasing range. Again, the changes in sign of the slopes for different common outcomes $(z, v)$ are violations of branch independence. This pattern is similar to that observed with three-outcome gambles by Birnbaum and Beeghley (1997).

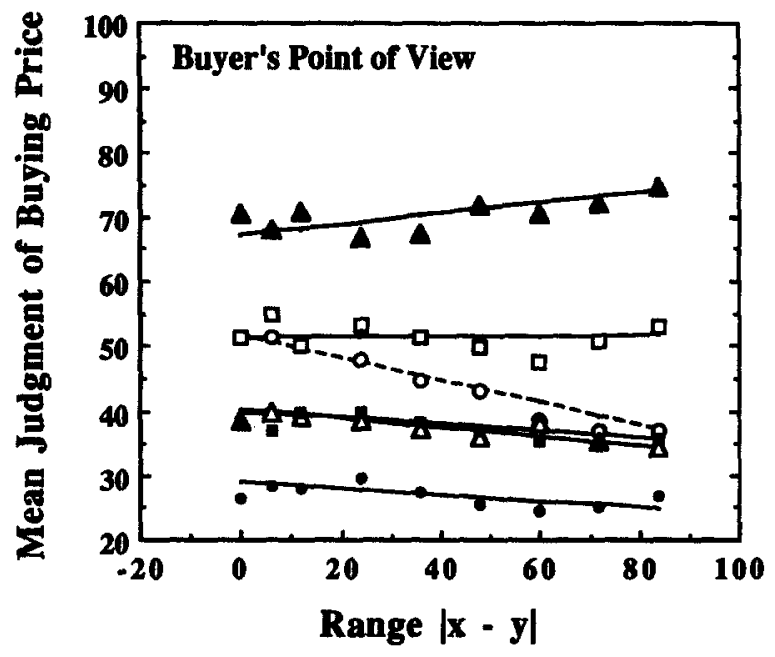

Figure 2. Judgments of the most that a buyer should pay for each gamble, for gambles in which $x+y=108$, plotted as a function of $|x-y|$, with separate curves for each pair of $z$ and $v$. Open circles show means for two-outcome gambles; dashed lines show corresponding predictions. Filled circles, open triangles, filled squares, open squares, and filled triangles show mean judgments for $(z, v)=$ $(\$ 2, \$ 4),(\$ 34, \$ 37),(\$ 19, \$ 52),(\$ 2, \$ 148)$, and $(\$ 124, \$ 148)$, respectively. Solid lines show predictions of the configural weight model using weights of Table 2.

\section{Individual and Statistical Analyses}

To determine the extent to which individual judges exhibited the same changes in slope (violations of branch independence) as the means in Figures 2 and 3, we

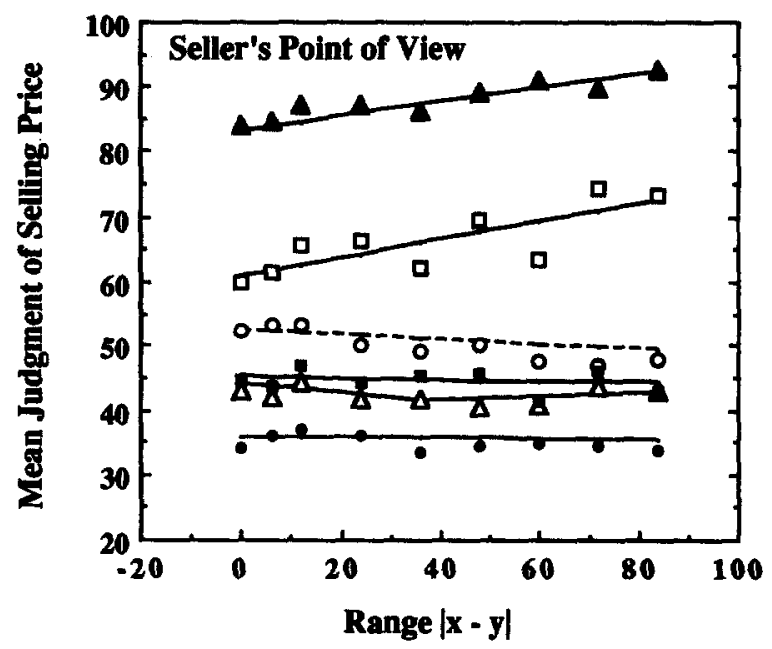

Figure 3. Judgments of the least that a seller should accept to sell each gamble, as a function of $|x-y|$ for gambles with $x+y=108$, with a separate curve for each pair of $z$ and $v$, as in Figure 2. Open circles show means for two-outcome gambles; dashed lines show corresponding predictions. Filled circles, open triangles, filled squares, open squares, and filled triangles show mean judgments for $(z, v)=(\$ 2, \$ 4),(\$ 34, \$ 37),(\$ 19, \$ 52),(\$ 2, \$ 148)$, and $(\$ 124$, $\$ 148)$, respectively. Solid lines show predictions of the configural weight model using weights of Table 2 . 
computed the signs of the slopes for each judge in each point of view as follows: For gambles with $x+y=108$, we compared the average response to gambles with $|x-y|=$ $\$ 6, \$ 12$, and $\$ 18$ against the average response to gambles with $|x-y|=\$ 60, \$ 72$, and $\$ 84$. In the buyer's viewpoint, for two-outcome gambles (open circles in Figure 2), 56 of the 60 judges showed negative slopes as a function of $|x-y|, 3$ showed positive slopes, and 1 showed no change. In the seller's viewpoint (open circles in Figure 3), 39 judges showed negative slopes, 16 showed positive slopes, and 5 showed zero slopes.

When $(z, v)=(\$ 2, \$ 148)$, however, 39 of the judges in the seller's viewpoint showed positive slopes, 18 showed negative slopes, and 3 showed zero slopes. The majority of individuals were consistent with the open squares in Figure 3. When these extreme common outcomes were added, 26 judges changed slope from negative to positive, compared with only 5 judges who changed in the opposite direction. In the buyer's viewpoint, 34 judges continued to have negative slopes when $(z, v)=(\$ 2, \$ 148)$, but 19 judges changed slopes from negative to positive, and none changed in the opposite direction. These two contrasts are statistically significant, $p<.01$, by binomial sign tests against the hypothesis that changes in slope should have split equally in either direction. Therefore, significantly more individuals changed in the directions shown in Figures 2 and 3 than in the opposite direction.

The change from the buyer's to the seller's viewpoint when $(z, v)=(\$ 2, \$ 148)$ caused 19 judges to change slopes from negative to positive, compared with only 3 who changed in the opposite direction (also significant, by sign test).

From the buyer's point of view, when $(z, v)=(\$ 2, \$ 4), 36$ judges showed negative slopes (consistent with greater weight on the medium-high outcome compared with the highest), 22 showed the opposite trend, and 2 showed no difference. From the seller's point of view, 38 participants showed negative slopes, 19 showed positive slopes, and 3 showed no change.

When the common pair was $(\$ 124, \$ 148)$, however, the majority in both points of view showed positive slopes. From the buyer's viewpoint, 34 judges showed positive slopes, compared with 24 judges who showed negative slopes. From the seller's viewpoint, 39 judges showed positive slopes, compared with 17 judges who showed negative slopes. In the buyer's point of view, 24 judges changed slopes from negative to positive, as $(z, v)$ changed from $(\$ 2, \$ 4)$ to $(\$ 124, \$ 148)$, and 13 judges changed in the opposite direction. In the seller's viewpoint, 29 judges changed from negative to positive and 8 changed in the opposite direction, a significant split. In sum, both points of view showed similar violations of branch independence when the common outcomes were changed from lowest to highest. This pattern is consistent with the assumption that weights of medium stimuli are greater than weights of extreme stimuli in both points of view, assuming $u(x)=x$.

For intermediate values of $(z, v)$, most people in both viewpoints showed negative slopes. For $(z, v)=(\$ 34, \$ 37)$ and $(\$ 19, \$ 52)$ in the buyer's viewpoint, 48 and 37 judges showed negative slopes against 10 and 19 judges who showed positive slopes, respectively. In the seller's view- point, 40 and 35 judges showed negative slopes against 20 and 24 judges who showed positive ones, respectively.

Analysis of variance of all of the judgments indicated that all main effects and interactions were statistically significant, including the main effect of point of view, $F(1,59)=$ 55.14 ; interaction of point of view and the common pair $(z$, $v), F(5,295)=34.00$; interaction of point of view and the variable pair $(x, y), F(27,1,593)=11.12$; interaction of the common pair and the $(x, y)$ Pair, $F(135,7,965)=11.74$; and the three-way interaction of Point of View $\times(z, v)$ Pair $\times$ $(x, y)$ Pair, $F(135,7,965)=2.55$.

These effects were also significant when the analyses were restricted to the cases in which $x+y=108$ (i.e., the data plotted in Figures 2 and 3). The slopes in Figures 2 and 3 represent the effect of the range of outcomes $x$ and $y$. The nonparallelism of the curves, averaged over Figures 2 and 3 , is significant, $F(40,2,360)=8.22$. The effect of range depends on the point of view, $F(8,472)=10.75$; the effect of common outcomes depends on the point of view, $F(5$, $295)=29.95$. The interaction between Range $\times$ Common Outcome also depends on point of view, $F(40,2,360)=$ 2.43. In sum, the slopes in Figures 2 and 3 show significant violations of branch independence, and these are significantly different in the different points of view.

These data also replicate a pattern of violation of branch independence that was observed in previous research (Birnbaum \& Beeghley, 1997, Figures 1 and 2). For example, we plotted judgments of buyer's prices for the wide-range gambles, $(z, v, \$ 12, \$ 84)$ and $(z, v, \$ 12, \$ 96)$, against the midpoint of $z$ and $v$ and compared them with mean judgments of gambles containing the low-range pair, $(z, v$, $\$ 39, \$ 45)$. The wide-range gambles crossed through the narrow range gambles such that the judgments of $(z, v, \$ 12$, $\$ 84)$ and $(z, v, \$ 12, \$ 96)$ are less than judgments of $(z, v, \$ 39$, $\$ 45)$ when the midpoint of the common outcomes is less than $\$ 40$, but judgments of $(z, v, \$ 12, \$ 84)$ and $(z, v, \$ 12$, $\$ 96)$ are greater than judgments of $(z, v, \$ 39, \$ 45)$ when the midpoint of the commons exceeded $\$ 75$. These crossing curves, like the changes in sign of the slopes in Figures 2 and 3 , are violations of branch independence.

\section{Estimation of Weights}

Equation 4 was fit to the data, predicting mean judgments from the lowest, medium-low, medium-high, and highest outcomes in each four-outcome gamble, separately for each point of view and separately for different combinations of common outcomes. Two-outcome gambles were also fit separately in each point of view. It was assumed that $u(x)=$ $x$, and $J$ was approximated as a linear function with an additive constant $(k)$, following Birnbaum et al. (1992).

Table 1 shows the estimated weights of the lower and higher outcomes in two-outcome gambles for the buyer's and the seller's point of view. Consistent with previous results (Birnbaum \& Beeghley, 1997; Birnbaum et al, 1992; Birnbaum \& Sutton, 1992) was the finding that the low outcome receives greater weight than the high outcome in the buyer's point of view. Also consistent with previous results was the finding that sellers place relatively more weight on the higher outcome and less weight on the lower 
Table 1

Estimated Parameters of Two Equally Likely Outcomes

\begin{tabular}{ccccc}
\hline & \multicolumn{4}{c}{ Point of view } \\
\cline { 2 - 5 } Parameter & \multicolumn{2}{c}{ Buyer's } & & \multicolumn{2}{c}{ Seller's } \\
\cline { 2 - 5 } \cline { 4 - 5 } & weight & $S E$ & weight & $S E$ \\
\hline$w_{\mathrm{L}}$ & 0.630 & .02 & 0.500 & .02 \\
$w_{\mathrm{H}}$ & 0.290 & .01 & 0.430 & .01 \\
$k$ & 1.540 & .76 & 1.540 & .92 \\
$R$ & 0.994 & & 0.993 & \\
Sum & 0.920 & & 0.933 & \\
\hline
\end{tabular}

Note. $S E$ refers to standard error of estimated parameters; $w_{\mathrm{L}}$ and $w_{\mathrm{H}}$ are the weights of lower and higher outcomes, respectively; $k$ is the additive constant; $R$ is the correlation between predicted and obtained mean judgments; sum designates sum of weights.

outcome than do buyers. From the seller's point of view, the weights of higher and lower outcomes are more nearly equal, but the low outcome still has slightly more weight than the high, unlike the results of Birnbaum et al. and Birnbaum and Stegner (1979).

The two rightmost columns of Table 2 show the best-fit weights, estimated from all 140 four-outcome gambles separately in each point of view. Weights of outcomes at the lower two ranks are greater in the buyer's point of view than in the seller's point of view. Similarly, weights for the two higher ranks are greater in the seller's point of view than in the buyer's point of view. Also, within each point of view, extreme outcomes receive less weight than their adjacent intermediate valued outcomes. These weights are plotted in Figure 4 for comparison with Figure 1.

Tables 1 and 2 show that the sum of the weights is greater for two-outcome gambles (0.92 and 0.93 for buyer's and seller's, respectively) than for four-outcome gambles ( 0.65 and 0.70 , respectively). Similarly, the sum of the weights of two lower outcomes (in a set of four) is less than the weight of one lower outcome in a set of two. However, the sum of weights of two higher outcomes in a set of four is about

\section{A. Buyer's Point of View}

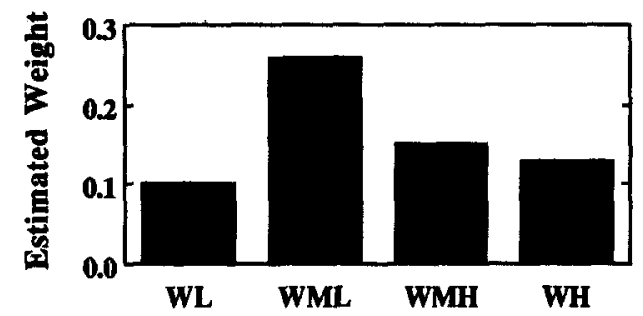

B. Seller's Point of View

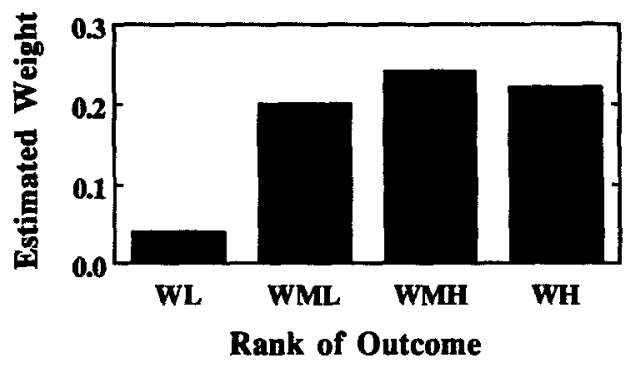

Figure 4. Estimated weights as a function of rank in each viewpoint. (A) Weights estimated in the buyer's viewpoint. (B) Weights estimated in the seller's viewpoint. WL, WML, WMH, and WH are the configural weights of the lowest, medium-low, medium-high, and highest outcomes, respectively.

equal to the weight of a single higher outcome in a set of two.

To check for higher order configural effects, we also estimated weights separately for each of four situations of $(z, v)$ outcomes in which these outcomes were least $(\$ 2, \$ 4)$, most $(\$ 124, \$ 148)$, extremes $(\$ 2, \$ 148)$, and intermediate $[(\$ 34, \$ 37)$ or $(\$ 19, \$ 51)]$ among $(x, y)$. Table 2 shows the best-fit weights for four-outcome gambles, estimated separately in these four situations. Weights for outcomes at the higher two ranks are higher in the seller's point of view than

Table 2

Weights of Four Equally Likely Outcomes Estimated Separately for Different $(z, v)$ Pairs

\begin{tabular}{|c|c|c|c|c|c|c|c|c|c|c|}
\hline \multirow[b]{2}{*}{ Parameter } & \multicolumn{2}{|c|}{$(\$ 2, \$ 4)$} & \multicolumn{2}{|c|}{$(\$ 124, \$ 148)$} & \multicolumn{2}{|c|}{$(\$ 2, \$ 148)$} & \multicolumn{2}{|c|}{$(\$ 34, \$ 37) \&(\$ 19, \$ 51)$} & \multicolumn{2}{|c|}{ All $(z, v)$} \\
\hline & $\begin{array}{c}\text { Buyer } \\
\text { weight } \\
(S E)\end{array}$ & $\begin{array}{c}\text { Seller } \\
\text { weight } \\
(S E)\end{array}$ & $\begin{array}{c}\text { Buyer } \\
\text { weight } \\
(S E)\end{array}$ & $\begin{array}{c}\text { Seller } \\
\text { weight } \\
(S E)\end{array}$ & $\begin{array}{c}\text { Buyer } \\
\text { weight } \\
(S E)\end{array}$ & $\begin{array}{c}\text { Seller } \\
\text { weight } \\
(S E)\end{array}$ & $\begin{array}{c}\text { Buyer } \\
\text { weight } \\
(S E)\end{array}$ & $\begin{array}{c}\text { Seller } \\
\text { weight } \\
(S E)\end{array}$ & $\begin{array}{c}\text { Buyer } \\
\text { weight } \\
(S E)\end{array}$ & $\begin{array}{c}\text { Seller } \\
\text { weight } \\
\text { (SE) }\end{array}$ \\
\hline$w_{\mathrm{L}}$ & - & - & 3) & 3) & - & - & 2) & 0.13 & $0.02)$ & 0.04 \\
\hline$w_{\mathrm{ML}}$ & - & - & $0.30(0.02)$ & $0.28(0.02)$ & $0.23(0.02)$ & $0.05(0.05)$ & 0.27 & 02) & $0.01)$ & $.02)$ \\
\hline$w_{\mathrm{MH}}$ & $0.28(0.02)$ & $0.31(0.02)$ & - & - & $0.24(0.01)$ & $0.33(0.03)$ & $0.19(0.02)$ & $0.34(0.02)$ & $0.15(0.01)$ & $0.24(0.01)$ \\
\hline$w_{H}$ & $0.18(0.01)$ & $0.30(0.01)$ & - & - & - & - & $0.12(0.01)$ & $0.18(0.01)$ & $0.13(0.01)$ & $0.22(0.01)$ \\
\hline$k$ & $4.2(0.80)$ & $3.1(0.94)$ & $43.6(1.3)$ & $64.3(1.2)$ & $26.0(1.0)$ & $40.3(2.2)$ & $4.75(0.90)$ & $4.18(0.92)$ & $8.69(0.45)$ & $7.98(0.57)$ \\
\hline$R$ & .976 & .983 & .962 & .959 & .968 & .910 & .977 & .983 & .991 & .992 \\
\hline Sum & .459 & .604 & .435 & .359 & .466 & .481 & .816 & .852 & .648 & .699 \\
\hline
\end{tabular}

Note. Parameters are estimated separately for 28 gambles with $(z, v)=(\$ 2, \$ 4),(\$ 124, \$ 148)$, and $(\$ 2, \$ 148)$, respectively. Estimates in the next two columns are based on 56 gambles with intermediate $(z, v)$ pairs. The last two columns show results for all 140 four-outcome gambles. Standard errors of parameters are shown in parentheses. $R$ is the correlation between predicted and obtained mean judgments within trials having fixed common outcomes. $w_{\mathrm{L}}, w_{\mathrm{ML}}, w_{\mathrm{MH}}$, and $w_{\mathrm{H}}$ are the weights of lowest, medium-low, medium-high, and highest outcomes, respectively. $k$ is the additive constant. Sum designates sum of weights. 
in the buyer's in all such comparisons in Table 2. Also, the medium-low rank always had greater estimated weight than the lowest rank, and the medium-high rank always had a greater weight than did the rank of the highest outcome, within both points of view. Thus, although the estimated weights show differences for different $(z, v)$ pairs, the separate weights show the same properties as the overall weights: Extreme outcomes receive less weight than adjacent intermediate outcomes within viewpoints; between viewpoints, sellers place more weight on the two higher ranks than do buyers, and buyers place more weight on the two lower ranks than do sellers.

For comparison, Birnbaum and Beeghley (1997) found estimated weights in the buyer's viewpoint were $w_{\mathrm{L}}=0.47$, $w_{\mathrm{M}}=0.30$, and $w_{\mathrm{H}}=0.07$. In the seller's viewpoint, the weights were $w_{\mathrm{L}}=0.23, w_{\mathrm{M}}=0.44$, and $w_{\mathrm{H}}=0.18$. (Note that the sums of these weights are 0.84 and 0.85 , respectively, also expressed in units of dollars, which are intermediate between the sums for two and four outcomes in the present experiment.) Birnbaum and Beeghley's weights show that in the buyer's viewpoint, judges placed most weight on the lowest value, whereas in the seller's viewpoint, judges placed most weight on the middle value. Also, two equal outcomes appear to have less weight than two unequal ones (Figs. 2 and 3 ) in both studies.

\section{Predicting Violations of Branch Independence}

The rank-dependent model correlates above .99 with the mean judgments for both two- and four-outcome gambles in both points of view (Table 1 and the rightmost columns of Table 2). However, correlations of fit can be high despite systematic deviations (Birnbaum, 1973); therefore, a more precise assessment of the ability of the rank-dependent model to predict violations of branch independence was made. First, the rank orders of the mean judgments were determined within each matrix of $28(x, y)$ pairs in each of the 6 sets of common outcomes. Then, a matrix of differences in rank order was calculated between each of the 15 pairs of 6 common outcomes (treating two-outcome gambles as another level of common outcomes), taken two at a time. We calculated corresponding matrices of differences in predicted rank order using the weights in Table 1 and the separately estimated weights in the left columns of Table 2. The variances of these rank-order differences were calculated within each matrix, and the correlations between changes in rank order between the predicted and obtained matrices were also calculated.

According to EU and SEU theories (Equation 1), there should be no violations of branch independence in this experiment. Because any change in rank order between matrices of common outcomes is a violation of branch independence, these theories imply that there should be no differences between matrices in the rank order of the $(x, y)$ pairs, except for random error. Therefore, SEU requires that any changes in rank order that are due to changes in the common outcomes should be small (variances should be small) and unpredictable. According to configural weight theory (Equation 3), the weights in Tables 1 and 2 imply that there should be differences in rank order and that these should be predictable from differences in rank order of the predictions.

The correlations between the 15 obtained and predicted variances of changes in rank order were significant for the buyer's and the seller's prices, respectively, .82 and .72 $(p<.01)$. The pooled correlations between predicted and obtained changes in rank order [pooled over 420 cells composed of 15 pairs of common outcomes by $28(x, y)$ pairs] were also significant, .66 and .52 for the buyer's and the seller's judgments, respectively. Any theory that requires branch independence is thus contradicted by the systematic changes in rank order that are predictable from the configural weights. These findings support those of Birnbaum and Beeghley (1997) for three-outcome gambles.

\section{Discussion}

Branch independence is systematically violated in judgments of two- and four-outcome gambles. The violations are consistent with the pattern reported by Birnbaum and Beeghley (1997) using three-outcome gambles. The present study found that intermediate stimuli have greater weight than extreme stimuli in both points of view. Thus, the case of three outcomes does not appear to be a unique case, as the use of four equally likely outcomes does not produce a qualitative change in the pattern of violations of branch independence.

\section{Violations of Branch Independence Contradict SEU}

Because branch independence is a weaker version of the sure thing principle of Savage (1954), violations of this property are a stronger refutation of SEU theory than is evidence with the Allais paradox, which remains consistent with the subjective weighted version of SEU, or the Ellsberg paradox (Ellsberg, 1961), which is a test of the stronger form of Savage's axiom applied to ambiguous situations (Stevenson, Busemeyer, \& Naylor, 1991).

The results in Figures 2 and 3 are not compatible with SEU, even allowing different utility functions for different points of view. Instead, results are consistent with configural weighting (Equation 4) and the premise that configural weights depend on point of view (Birnbaum et al., 1992; Birnbaum \& Stegner, 1979; Birnbaum \& Sutton, 1992). This theory accounts for the different rank orders of judgments in the two points of view, for violations of branch independence, and for changes in violation of branch independence between the two points of view.

\section{Similarities and Differences Between Judgment and Choice}

The present results are based on judgments of gambles rather than choices between gambles. Judgment and choice experiments do not always agree, and there are several situations in which different preference orders require complex interpretations (Mellers, Ordóñez, \& Birnbaum, 1992; Birnbaum \& Sutton, 1992). However, the pattern of violations of branch independence observed in the present 
judgment tasks, characterized by Expressions 5a-5b, has also been observed in a recent choice experiment by Birnbaum and Chavez (1997). Birnbaum and Chavez used choices between three- and four-outcome gambles in which probabilities were specified. Violations of branch independence in choices between four-outcome gambles were consistent with greater weight on intermediate stimuli than on extremes. Results also appear compatible with the same utility function for choice as for judgment, $u(x)=x$, for $0<$ $x<\$ 150$.

Similarly, with three equally likely outcomes, judgments of buying prices, selling prices, and choices have quite different rank orders but can be fit with the same utility function, using different configural weights that satisfy Expression 7 (Birnbaum \& Beeghley, 1997). Similar to Expression 5b, Expression 7 also assigns greater weight to the middle outcome than to (the geometric average of) the extremes. Birnbaum and McIntosh (1996) fit a configuralweight model to their choice results and estimated relative weights of $w_{\mathrm{L}}=0.51, w_{\mathrm{M}}=0.33$, and $w_{\mathrm{H}}=0.16$. The relative weights for the buyer's point of view in Birnbaum and Beeghley (1997) were $w_{\mathrm{L}}=0.56, w_{\mathrm{M}}=0.36$, and $w_{\mathrm{H}}=$ 0.08 ; for the seller's point of view, the relative weights were $w_{\mathrm{L}}=0.27, w_{\mathrm{M}}=0.52$, and $w_{\mathrm{H}}=0.21$. Weights for choice appear to be intermediate between the two points of view in judgment and closer to the buyer's than to the seller's point of view.

The present results for judgments of two-outcome gambles (Table 1) also appear consistent with experiments using judgments of strength of preference between gambles composed of two equally likely outcomes; tests of interval independence imply that lower outcomes receive greater weight (Birnbaum, Thompson, \& Bean, 1997). The present results are also compatible with judgment results of Birnbaum et al. (1992) and of Birnbaum and Sutton (1992). Experiments with two-outcome gambles thus appear to contradict the theory of Quiggin (1982), which implies that two equally likely outcomes should receive equal weight; instead, in judgments from the buyer's and the neutral's perspective and judgments of strength of preference, lower outcomes tend to receive greater weight.

The representation of viewpoint in the configural weight model assumes that there are many points of view on a continuum from that of a very cautious buyer to that of a very venturesome seller. By changing instructions (e.g., "Your task is to advise a cautious seller who is willing to sell low rather than accept a risk of loss"), it should be possible to produce a viewpoint such that the higher and lower outcomes receive more nearly equal weight or to produce more extreme patterns of weighting (e.g., "Advise a venturesome seller who would really hate to have sold a gamble that pays off"). Perhaps with such a change in instructions, it would be possible to create a viewpoint in which a seller would assign greatest weight to the highest stimulus.

As noted by Birnbaum (1974a), the configural weight model can vary from a minimum model, in which the response depends entirely on the lowest outcome, to a maximum model, in which the response depends entirely on the highest outcome (see also Birnbaum et al., 1992;
Birnbaum \& Sotoodeh, 1991; Birnbaum \& Stegner, 1979; Birnbaum \& Sutton, 1992). It should not be regarded as an anomaly when a buyer refuses to pay more than the lowest outcome or when the seller refuses to sell for less than the highest outcome. Such behavior may seem inconsistent with EU theory, but it is compatible with configural weight theory.

\section{Results: Opposite of Inverse-S Weighting in Cumulative Prospect Theory}

The patterns in Figures 2 and 3 are not consistent with the inverse-S weighting function used in cumulative prospect theory (Tversky \& Kahneman, 1992). To fit median certainty equivalents of two-outcome gambles as a function of probability, Tversky and Kahneman used a weighting function that is flatter in the middle. Assuming Expression 8a, this function assigns lower weights to the two medium outcomes in a set of four equally likely outcomes, and it therefore predicts the opposite pattern of violation from the pattern observed in the experiment.

The inverse-S weighting function incorrectly predicts that the curves for $(z, v)=(\$ 2, \$ 4)$ in Figures 2 and 3 should have positive slope as a function of range because the weight of the middle-high outcome is supposed to be less than that of the highest outcome. The positive slopes in Figures 2 and 3 for $(z, v)=(\$ 124, \$ 128)$ are also inconsistent with the cumulative prospect model because the medium-low outcome is supposed to have less weight than the lowest outcome. Similarly, the patterns of violation of branch independence in Birnbaum and Beeghley (1997), the choice data of Birnbaum and McIntosh (1996), and choice results of Birnbaum and Chavez (1997) are opposite the pattern predicted by the inverse-S weighting function of cumulative prospect theory.

The power function (Expression 8c) suggested as a possible cumulative weighting function by Luce and Fishburn $(1991,1995)$ implies that weights are a monotonic function of rank, which our estimated weights are not. Instead, weights appear greatest for intermediate level stimuli, inferred from the opposite sign slopes in Figures 2 and 3. These changing slopes indicate that if $u(x)=x$, then the intermediate ranks have greater weight than the extremes in both points of view. For this weighting function to be consistent with the present data, it would need a different utility function.

The finding that intermediate stimuli have greater weight than extreme stimuli seems consistent with weighting schemes used in the scoring of certain athletic events, such as diving, where the highest and lowest marks are thrown out and the remaining scores are averaged. Such a weighting pattern also seems consistent with judgment data obtained in the study of range-frequency theory. When asked to select a typical or average number to represent a distribution of numbers, judges select a value that is a weighted compromise between the midpoint and median on the subjective scale (Birnbaum, 1974b; Parducci, 1963, 1995). The median of four outcomes could be obtained by throwing out the two extremes and averaging the two medium stimuli. Range- 
frequency theory by itself does not, however, explain the greater weighting on lower-valued outcomes or the difference between buying and selling prices.

The weighting pattern would be consistent with cumulative prospect theory (Expression 8a) and $u(x)=x$ if the decumulative weighting function is $S$-shaped rather than inverse-S shaped. This apparent contradiction between the inverse-S of Tversky and Kahneman (1992) and $\mathrm{Wu}$ and Gonzalez (1996) and the present results (and those of Birnbaum \& Beeghley, 1997; Birnbaum \& Chavez, 1997; Birnbaum \& McIntosh, 1996) may be a contradiction only within cumulative prospect theory; there is no contradiction in configural weight theory. The model of Birnbaum and McIntosh can describe the results of Tversky and Kahneman and of Wu and Gonzalez without changing parameters.

We suspect that cumulative prospect theory can be improved by giving up the decumulative weighting function (Expression 8a). The attraction of cumulative prospect theory over original prospect theory is that it incorporates rank dependence without violating stochastic dominance (Tversky \& Kahneman, 1992). It is an empirical question whether stochastic dominance is satisfied or if it is systematically violated (Birnbaum, 1992a, 1992b, 1997; Birnbaum et al., 1992; Birnbaum \& Thompson, 1996; Tversky \& Kahneman, 1986; von Winterfeldt, Chung, Luce, \& Cho, 1997).

\section{Testing Cumulative Prospect Theory}

Birnbaum (1997) deduced two cumulative independence conditions that must be satisfied by cumulative prospect theory with any weighting function; these conditions are a combination of transitivity, monotonicity, comonotonic independence, and a coalescing equivalence. Because the present experiment does not directly test those assumptions, it does not by itself contradict the core of cumulative prospect theory (Equations 3 and $8 \mathrm{a}$ ). The present study does require a weighting function that contradicts the particular inverse-S weighting function, assuming any utility function.

Wakker et al. (1994) conducted a study of choices, comparing rates of comonotonic and noncomonotonic independence. They did not find sufficient evidence to reject EU theory in favor of RDU theory. By increasing the range of outcomes used and making other modifications to the procedure in Wakker et al., Weber and Kirsner (1997) observed small but systematic violations of branch independence that appeared to be in agreement with RDU theory. The results of Weber and Kirsner are consistent with the present findings: As value of the common outcome(s) is increased, preference for gambles containing the wider ranged outcomes increased.

Wakker et al. (1994) concluded that tests of comonotonic independence are the key distinction between EU and RDU theories. That conclusion is certainly true for the standard RDU theory, but configural weight theory that is derived from asymmetric loss functions (Birnbaum et al., 1992; Birnbaum \& Mclntosh, 1996; Weber, 1994) can lead to different RDU representations in different subdomains because this model implies configural weights that depend on the spacing among the outcomes as well as their ranks.
Birnbaum and McIntosh (1996, Appendix A) have shown that minimization of the asymmetrically weighted squared loss function, with different weights for over and underestimation, leads to a configural weight model that violates comonotonic independence. Thus, the study of patterns of comonotonic independence will be an important key to distinguishing different configural weighting models.

\section{Conclusions}

According to SEU theories, people prefer sure wins over gambles with positive expected value because the utility function for money is concave downwards. However, the present results, along with judgment data of Birnbaum and Beeghley (1997) and choice data of Birnbaum and Chavez (1997) and Birnbaum and McIntosh (1996), are inconsistent with EU theories. Although violations of branch independence are inconsistent with SEU theory, they are consistent with RDU theories. RDU theory can account for risk aversion, preference reversals, and violations of branch independence, using a linear utility function, $u(x)=x$, if outcomes of lower rank have greater weight.

Although buying and selling prices are not monotonically related, the different preference orders can be fit by the assumptions that the utility function is invariant with respect to point of view and that only configural weights change between viewpoints. The change in weights explains different violations of branch independence in different viewpoints. Although weights are different in different viewpoints, they have a property in common among buying prices, selling prices, and choices: The ratio of weights of the medium-high to that of the highest outcome exceeds the ratio of weights of lowest to medium-low outcome. This property produces a pattern of violations of branch independence that is observed in both points of view but that is opposite the pattern predicted by the inverse-S weighting function of cumulative prospect theory.

\section{References}

Birnbaum, M. H. (1973). Morality judgment: Test of an averaging model with differential weights. Journal of Experimental Psychology, 99, 395-399.

Birnbaum, M. H. (1974a). The nonadditivity of personality impressions. Journal of Experimental Psychology, 102, 543-561.

Birnbaum, M. H. (1974b). Using contextual effects to derive psychophysical scales. Perception \& Psychophysics, 15, 89-96.

Birnbaum, M. H. (1982). Controversies in psychological measurement. In B. Wegener (Ed.), Social attitudes and psychophysical measurement (pp. 401-485). Hillsdale, NJ: Erlbaum.

Birnbaum, M. H. (1992a). Issues in utility measurement. Organizational Behavior and Human Decision Processes, 52, 319-330.

Birnbaum, M. H. (1992b). Violations of monotonicity and contextual effects in choice-based certainty equivalents. Psychological Science, 3, 310-314.

Birnbaum, M. H. (1997). Violations of monotonicity in judgment and decision making. In A. A. J. Marley (Ed.), Choice, decision and measurement: Essays in honor of $R$. Duncan Luce (pp. 73-100). Mahwah, NJ: Erlbaum. 
Birnbaum, M. H., \& Beeghley, D. (1997). Violations of branch independence in judgments of the value of gambles. Psychological Science, 8, 87-94.

Birmbaum, M. H., \& Chavez, A. (1997). Tests of theories of decision making: Violations of branch independence and distribution independence. Organizational Behavior and Human Decision Processes, 71, 161-194.

Birnbaum, M. H., Coffey, G., Mellers, B. A., \& Weiss, R. (1992). Utility measurement: Configural-weight theory and the judge's point of view. Journal of Experimental Psychology: Human Perception and Performance, 18, 331-346.

Birnbaum, M. H., \& McIntosh, W. R. (1996). Violations of branch independence in choices between gambles. Organizational Behavior and Human Decision Processes, 67, 91-110.

Birnbaum, M. H., \& Sotoodeh, Y. (1991). Measurement of stress: Scaling the magnitudes of life changes. Psychological Science, 2, 236-243.

Birnbaum, M. H., \& Stegner, S. E. (1979). Source credibility in social judgment: Bias, expertise, and the judge's point of view. Journal of Personality and Social Psychology, 37, 48-74.

Birnbaum, M. H., \& Stegner, S. E. (1981). Measuring the importance of cues in judgment for individuals: Subjective theories of IQ as a function of heredity and environment. Journal of Experimental Social Psychology, 17, 159-182.

Birnbaum, M. H., \& Sutton, S. E. (1992). Scale convergence and utility measurement. Organizational Behavior and Human Decision Processes, 52, 183-215.

Birnbaum, M. H., \& Thompson, L. A. (1996). Violations of monotonicity in choices between gambles and certain cash. American Journal of Psychology, 109, 501-523.

Birnbaum, M. H., Thompson, L. A., \& Bean, D. J. (1997). Testing interval independence versus configural weighting using judgments of strength of preference. Journal of Experimental Psychology: Human Perception and Performance, 23, 939-947.

Birnbaum, M. H., \& Zimmermann, J. (1995, February). The Savage Memorial Lecture: Exploring the mind of Bob Citron. Paper presented at the Bayesian Research Conference, Studio City, CA.

Champagne, M., \& Stevenson, M. K. (1994). Contrasting models of appraisal judgments for positive and negative purposes using policy modeling. Organizational Behavior and Human Decision Processes, 59, 93-123.

Edwards, W. (1954). The theory of decision making. Psychological Bulletin, 51, 380-417.

Edwards, W. (Ed.). (1992). Utility theories: Measurements and applications. Boston: Kluwer Academic.

Ellsberg, D. (1961). Risk, ambiguity and the Savage axioms. Quarterly Journal of Economics, 75, 643-649.

Kahneman, D., \& Tversky, A. (1979). Prospect theory: An analysis of decision under risk. Econometrica, 47, 263-291.

Krantz, D. H., Luce, D., Suppes, P., \& Tversky, A. (1971). Foundations of measurement. New York: Academic Press.

Lopes, L. (1990). Re-modeling risk aversion: A comparison of Bernoullian and rank dependent value approaches. In G. M. v. Furstenberg (Ed.), Acting under uncertainty (pp. 267-299). Boston: Kluwer Academic.

Luce, R. D. (1992). Where does subjective expected utility fail descriptively? Journal of Risk and Uncertainty, 5, 5-27.

Luce, R. D., \& Fishburn, P. C. (1991). Rank- and sign-dependent linear utility models for finite first order gambles. Journal of Risk and Uncertainty, 4, 29-59.

Luce, R. D., \& Fishburn, P. C. (1995). A note on deriving rank-dependent utility using additive joint receipts. Joumal of Risk and Uncertainty, 11, 5-16.

Luce, R. D., \& Narens, L. (1985). Classification of concatenation measurement structures according to scale type. Journal of Mathematical Psychology, 29, 1-72.

Machina, M. J. (1982). Expected utility analysis without the independence axiom. Econometrica, 50, 277-323.

Mellers, B. A., Ordóñez, L., \& Birnbaum, M. H. (1992). A change-of-process theory for contextual effects and preference reversals in risky decision making. Organizational Behavior and Human Decision Processes, 52, 331-369.

Miyamoto, J. M. (1989), Generic utility theory: Measurement foundations and applications in multiattribute utility theory. Journal of Mathematical Psychology, 32, 357-404.

Parducci, A. (1963). The range-frequency compromise in judgment. Psychological Monographs, 77(2, Whole No. 565).

Parducci, A. (1995). Happiness, pleasure, and judgment. Mahwah, NJ: Erlbaum.

Quiggin, J. (1982). A theory of anticipated utility. Journal of Economic Behavior and Organization, 3, 324-345.

Savage, L. J. (1954). The foundations of statistics. New York: Wiley.

Schmeidler, D. (1989). Subjective probability and expected utility without additivity. Econometrica, 57, 571-587.

Stevenson, M. K., Busemeyer, J. R., \& Naylor, J. C. (1991). Judgment and decision-making theory. In M. Dunnette \& L. M. Hough (Eds.), New handbook of industrial-organizational psychology (pp. 283-374). Palo Alto, CA: Consulting Psychologist Press.

Tversky, A., \& Kahneman, D. (1986). Rational choice and the framing of decisions. Journal of Business, 59, S251-S278.

Tversky, A., \& Kahneman, D. (1992). Advances in prospect theory: Cumulative representation of uncertainty. Journal of Risk and Uncertainty, 5, 297-323.

Tversky, A., \& Wakker, P. (1995). Risk attitudes and decision weights. Econometrica, 63, 1255-1280.

yon Winterfeldt, D., Chung, N., Luce, R. D., \& Cho, Y. (1997). Tests of consequence monotonicity in decision making under uncertainty. Journal of Experimental Psychology: Learning, Memory, and Cognition, 23, 406-426.

Wakker, P. (1993). Additive representations on rank-ordered sets. II. The topological approach. Journal of Mathematical Economics, 22, 1-26.

Wakker, P. (1994). Separating marginal utility and probabilistic risk aversion. Theory and Decision, 36, 1-44.

Wakker, P., Erev, I., \& Weber, E. U. (1994). Comonotonic independence: The critical test between classical and rankdependent utility theories. Journal of Risk and Uncertainty, 9, 195-230.

Weber, E. U. (1994). From subjective probabilities to decision weights: The effects of asymmetric loss functions on the evaluation of uncertain outcomes and events. Psychological Bulletin, 114, 228-242.

Weber, E. U., Anderson, C. J., \& Birnbaum, M. H. (1992). A theory of perceived risk and attractiveness. Organizational Behavior and Human Decision Processes, 52, 492-523.

Weber, E. U., \& Kirsner, B. (1997). Reasons for rank-dependent utility evaluation. Journal of Risk and Uncertainty, 14, 41-61.

Wu, G., \& Gonzalez, R. (1996). Curvature of the probability weighting function. Management Science, 42, 1676-1690.

Yaari, M. E. (1987). The dual theory of choice under risk. Econometrica, 55, 95-115.

Received July 3, 1996

Revision received December 12, 1996

Accepted December 12, 1996 